\title{
Experimental investigation of transients in six cascaded AGC EDFAs and their suppression using a high-speed VOA
}

\author{
Kiyo Ishii ${ }^{1 a)}$, Junya Kurumida ${ }^{1}$, Ken Tanizawa ${ }^{1}$, Shu Namiki ${ }^{1}$, \\ Norihiko Sato $^{2}$, Kazuya Ota ${ }^{2}$, Yoichi Oikawa ${ }^{2}$, \\ and Noriyasu Shiga ${ }^{2}$ \\ ${ }^{1}$ National Institute of Advanced Industrial Science and Technology, Network Pho- \\ tonics Research Center, 1-1-1 Umezono, Central 2, Tsukuba, 305-8568, Japan \\ 2 Trimatiz Limited, B801 La Pacifique, 4-7-12 Minamiyawata, Ichikawa, Chiba \\ 272-0023 Japan \\ a)kiyo-ishii@aist.go.jp
}

Abstract: We investigate gain transient responses of an automatic gain control (AGC) EDFA with fast electrical feed-forward pump control. Even if each of the AGC EDFAs can well suppress the gain transients for add/drop operations with various switch speeds, the cumulative transients in cascaded AGC EDFAs give rise to a non-negligible impact. We demonstrate that a high-speed variable optical attenuator (VOA) can suppress such cumulative transients. Error-free operation of a surviving channel under the instantaneous load and unload condition with six cascaded AGC EDFAs is confirmed. The burst mode signal is 7-ms duration with 16 -dB range.

Keywords: EDFA transient suppression, optical fast circuit switching network

Classification: Fiber-Optic Transmission for Communications

\section{References}

[1] K. Okamura, E. Otani, T. Yoshikawa, T. Uchino, M. Fukushima, and N. Kagi, "Optical burst amplification using EDFA with fast feedback control," Proc. OFC/NFOEC 2005, Anaheim, USA, OTuN2, March 2005.

[2] Y. Awaji, H. Furukawa, N. Wada, P. Chan, and R. Man, "Mitigation of Transient Response of Erbium-Doped Fiber Amplifier for Burst Traffic of High Speed Optical Packets," Proc. QELS 200\%, Baltimore, USA, JTuA133, May 2007.

[3] G. Luo, L. Ayskind, Y. Sun, A. K. Srivastava, J. W. Sulhoff, C. Wolf, and A. Ali, "Performance Degradation of All-Optical Gain-Clamped EDFA's Due to Relaxation-Oscillations and Spectral-Hole Burning in Amplified WDM Network," IEEE Photon. Technol. Lett., vol. 9, no. 10, pp. 13461348, Oct. 1997. 
[4] S. Y. Park, H. K. Kim, G. Y. Lyu, S. M. Kang, and S.-Y. Shin, "Dynamic Gain and Output Power Control in a Gain-Flattened Erbium-Doped Fiber Amplifier," IEEE Photon. Technol. Lett., vol. 10, no. 6, pp. 787-789, June 1998.

[5] L. Rapp, "Transient Performance of Erbium-doped Fiber Amplifiers using a New Feedforward Control taking into account Wavelength," J. Opt. Commun., vol. 28, no. 2, pp. 82-90, June 2007.

[6] A. Lieu, C. Tian, and T. Naito, "Transmission and interactions of WDM burst signals in cascaded EDFAs," Proc. OFC/NFOEC 2006, Anaheim, USA, OTuD5, March 2006.

[7] M. Murakami, T. Seki, and K. Oda, "Optial channel power stability in transparent optical network using large-scale photonic crossconnects and automatic gain control EDFAs," Proc. 10th ICTON 2008, Athens, Greece, Tu.D1.6, June 2008.

[8] N. Sato, K. Ota, N. Mishima, Y. Oikawa, and N. Shiga, "Less than 0.19-dB Transient Gain Excursion AGC-EDFA with Digital Control for 20-Channel Add/Drop Equivalent Operation," Proc. OFC/NFOEC 2011, Los Angels, USA, OMH3, March 2011.

\section{Introduction}

Dynamical switching of optical signals in the optical domain receives increasing importance as a cut-through operation or protection/restoration in optical domain is highly demanded due to cost and energy pressure. Because fast and large changes of the input power levels to erbium-doped optical amplifiers (EDFAs) cause dynamic gain transients and hence deteriorate transmission quality, the conventional reconfigurable optical add/drop multiplexer (ROADM) systems have been significantly limited in its flexibility and scale as well as reconfiguration speed. In fact, even though several techniques have been proposed to suppress the transients of one-stage EDFAs [1, 2, 3, 4, 5], the cascaded operations of the EDFAs with such techniques would still suffer from severe cumulative transients in particular for high-speed switching operation $[6,7]$, which remains substantially unsolved to date to our knowledge. Fundamentally, no matter how fast and properly the pump power is controlled, the intrinsic dynamics of the erbium ions cannot be completely controlled externally.

On the other hand, a high-speed variable optical attenuator (VOA) with a response time of less than $1 \mu$ s has been adopted to suppress such remnant transients in conjunction with a fast electrical feed-forward pump control to achieve better suppression of gain excursions within much faster settling time [8]. A gain excursion of less than $0.19 \mathrm{~dB}$ per unit was realized by the AGC EDFA with a VOA for a 20-channel add/drop equivalent operation.

This study is an extension of [8] in particular to a cascaded operation. Firstly, we investigate the transients of the AGC EDFA for various optical switches. Secondly we prepare six AGC EDFAs with feed-forward pump control and one high-speed VOA for investigating their cascaded transient performance. We suppose to change the input power of the AGC EDFAs 
by $16 \mathrm{~dB}$ using an optical switch with nano-second order switch speed. We demonstrate that the high-speed VOA can suppress the accumulated transients to $0.8-2 \mathrm{~dB}$ and the bit error rate (BER) of a surviving channel stays error free. To the best of our knowledge, this is the best result of ever reported transient performances while the cascaded operation emulates one of the severest case in terms of the input power dynamic range and the add/drop switch speed.

\section{Transient response check for optical switches}

We prepared three kinds of optical switches that offer different switching speed each other in order to investigate the transient overshoot and undershoot of the single stage operation of the AGC EDFA without a high-speed VOA [8]. The experimental setup is shown in Fig. 1 (a). We input two wavelengths to the AGC EDFA, $1550.116 \mathrm{~nm}$ and $1550.918 \mathrm{~nm}$. The wavelength channel of $1550.116 \mathrm{~nm}$ is continuously input and we call this surviving channel, while the wavelength channel of $1550.918 \mathrm{~nm}$ is added or dropped using optical switches and we call this aggressive channel. The AGC EDFA input power dynamic range was set to $16 \mathrm{~dB}$ that corresponds to sudden add/drop of 39 channels in a 40-channel system. In other words, we emulated the largest input power change on a 40-channel WDM transmission in $100 \mathrm{GHz}$ ITU-T grid in C-band. Here we used the 2-channel scheme because our focus here is to investigate the EDFA transients due to input power changes. However, we note that evaluations in a full WDM scheme should be conducted to apply an EDFA to a WDM transmission system. This is our next target.

Fig. 1 (b) shows the measured waveform of fast optical switch whose $10 \%-$ $90 \%$ switching time is less than 70 ns, Fig. 1 (c) that of MEMS switch whose switching time is less than 500 us, and Fig. 1 (d) that of LCOS based WSS whose switching time is less than $40 \mathrm{~ms}$. All the measured transient over-

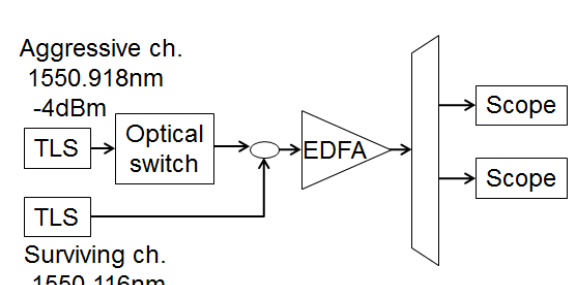

$1550.116 \mathrm{~nm}$

$-20 \mathrm{dBm}$

(a) Experimental setup

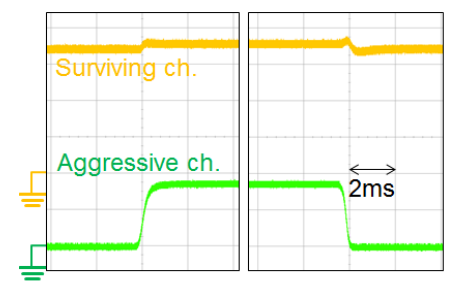

(c) MEMS switch

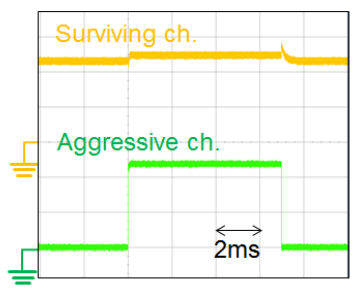

(b) Fast optical switch whose rise and fall times are less than 70ns

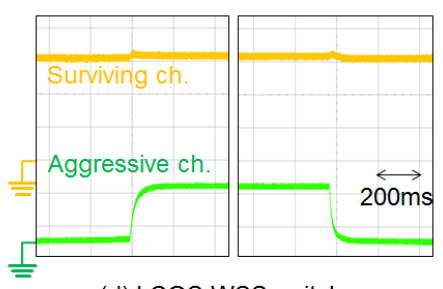

(d) LCOS WSS switch

Fig. 1. Experimental setup and measured waveforms for three kinds of optical switches 
shoots and undershoots are less than $0.5 \mathrm{~dB}$. They are well suppressed for single EDFA operation while the faster optical switch provides the larger transients.

\section{Cascaded operations with high-speed optical switch}

Fig. 2 (a) shows the experimental setup for a cascaded operation. We cascaded six feed-forward pump controlled AGC EDFAs. We used the fast optical switch for adding/dropping the aggressive channel in order to examine the severest case according to the experimental results shown in Sec. 2. The optical switch was driven by a function generator and generated an optical burst pulse with duration of $7 \mathrm{~ms}$ and a duty cycle of $7 \%$. We note that this condition is after [6] for reference. The input levels of the surviving and aggressive channels to each AGC EDFA were $-20 \mathrm{dBm}$ and $-4 \mathrm{dBm}$, respectively. However, we have slightly adjusted and optimized input power levels such that the transients were suppressed as much as possible. We load 10-Gbps NRZ $2^{31}-1$ pseudo-random bit sequence (PRBS) on the surviving channel in order to monitor its BER performance since the impact of add/drop of the aggressive channels is larger for the surviving channel than the aggressive channels themselves. The optical attenuators (ATTs) emulate either the span or node losses. The optical band-pass filters (BPFs) after EDFAs eliminate the ASE noise of EDFAs and the bandwidth is set to $2 \mathrm{~nm}$.

Fig. 2 (b) shows the cumulative transient overshoots and undershoots at each stage. The figure indicates that the transients tend to linearly accumulate in $\mathrm{dB}$, and that even small transients per stage will cumulatively give rise to a non-negligible impact at the receiver end. Fig. 2 (c) shows the measured waveform after the 6th EDFA. Due to the feed-forward control rather than feed-back control of pump lasers, we did not observe any oscillatory behavior

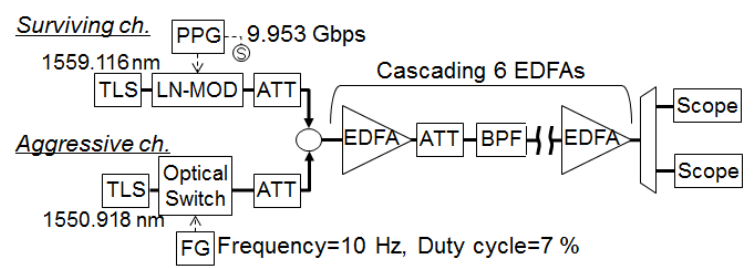

(a) Experimental setup

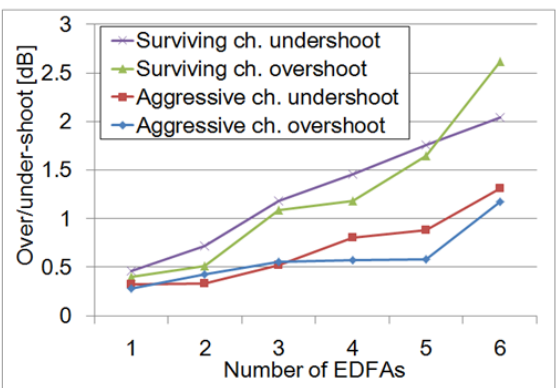

(b) Transients vs. number of EDFAs

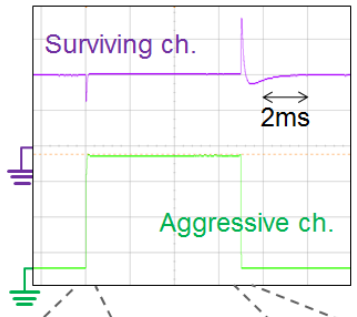

Magnified figure of Magnified' figurè of rising edge

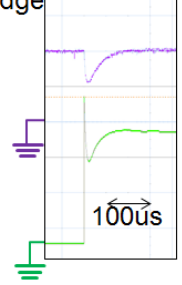

(c) Measured waveform after 6 EDFAs

Fig. 2. Experimental setup and measurements of cumulative transient effects 
as reported in [6].

\section{Suppression of cumulative transients using a high-speed VOA}

In order to suppress the cumulative transients, we inserted one high-speed VOA after the 6th EDFA of the experimental setup shown in Fig. 2 (a). The high-speed VOA can reduce both overshoot and undershoot. The detailed high-speed VOA operation is described in [8]. In the simple terms, the highspeed VOA loss is reduced in order to offset the undershoot when the burst signal is added and the high-speed VOA loss is increased in order to eliminate the overshoot when the burst signal is dropped. The measured waveforms show that the high-speed VOA can effectively suppress the overshoot of the surviving channel to less than $1 \mathrm{~dB}$ as shown in Fig. 3 (a). The undershoot of the surviving channel was still $1.9 \mathrm{~dB}$ however the suppression of the undershoot will be more improved by optimizing the high-speed VOA control parameters. The effects of transient suppression by high-speed VOA were also observed in eye diagrams shown in Fig. 3(b). Many spots are observed outside of the eye in Fig. 3 (b) without VOA case (left).

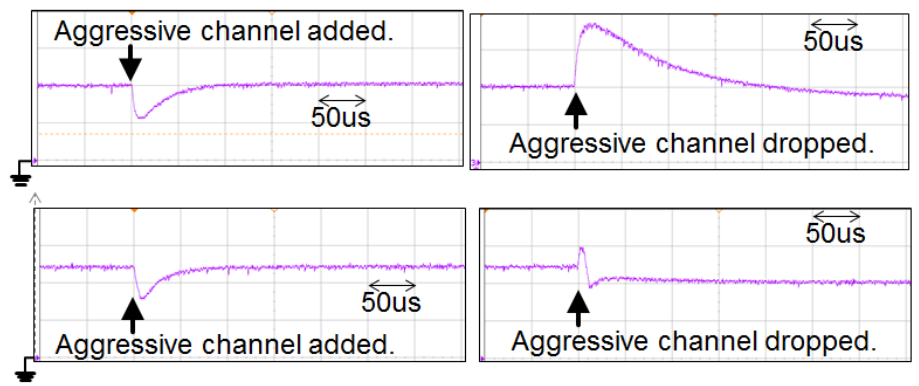

(a) Measured waveforms after 6 EDFAs w/o VOA (upper) and w/ VOA (lower)
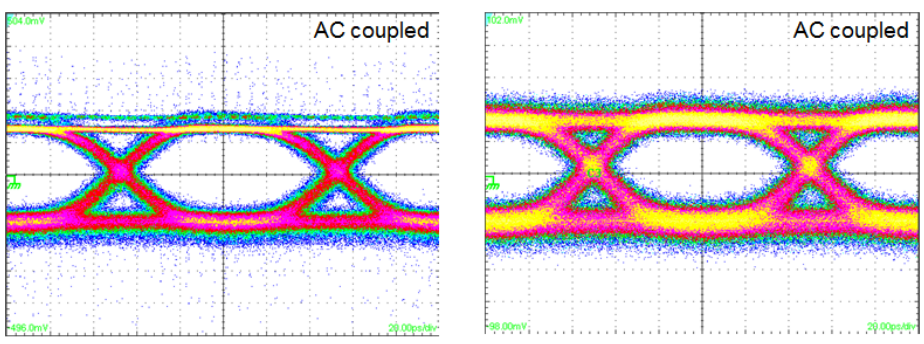

(b) Measured eye diagrams after 6 EDFAs w/o VOA (left) and w/ VOA (right)

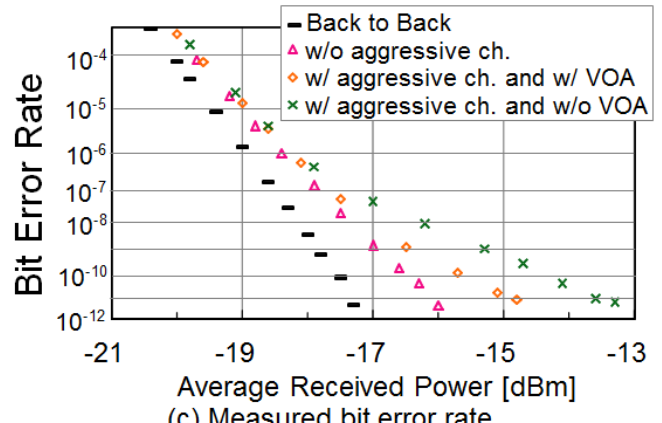

(c) Measured bit error rate

Fig. 3. Transient suppression by high-speed VOA after 6 EDFAs 
Fig. 3 (c) shows the measured BER characteristics. The power penalty at the BER of $10^{-9}$ without aggressive channel was $0.9 \mathrm{~dB}$, that with aggressive channel and without high-speed VOA was $2.3 \mathrm{~dB}$, and that with aggressive channel and high-speed VOA was $1.3 \mathrm{~dB}$. Consequently, the BER degradation due to the accumulation of the EDFA transients was $1.4 \mathrm{~dB}$ and inserting a high-speed VOA after 6 EDFAs can improve the power penalty by $1 \mathrm{~dB}$ in this experiment.

Even in the presence of the largest input power change in a 40-channel ROADM network, the six cascaded AGC EDFAs with electrical feed-forward pump control can provide a stable operation of the surviving channel with the aid of the high-speed VOA. We have confirmed that better algorithm to control the VOA would better suppress the overshoot and undershoot. The other alternative would be to deploy high-speed VOAs at each of the EDFAs that would suppress the accumulation of the transients.

\section{Conclusion}

In the paper, we have investigated transient responses of an AGC EDFA with electrical feed-forward pump control. Firstly we confirmed the AGC EDFA could well suppress the transients for a sudden input power change using optical switches with various switch speeds nevertheless we also confirmed faster switch speed imposed larger transients. Secondly, we experimentally demonstrated that even small transients per AGC EDFA cumulatively produced a non-negligible impact at the receiver end in cascaded operations. Finally, we demonstrated a high-speed VOA following the six cascaded AGC EDFAs could suppress the accumulated transients and improve the power penalty. Error-free operation of the surviving channel during instantaneous load and unload of a 7-ms burst signal with 16-dB dynamic range in the six cascaded AGC EDFAs was confirmed. To the best of our knowledge, this is the best result ever reported while we here emulated one of the severest case for EDFA transients in terms of the input power dynamic range and the switch speed of add/drop operations.

\section{Acknowledgments}

This work was partly supported by Project for Developing Innovation Systems of the Ministry of Education, Culture, Sports, Science and Technology (MEXT), Japan. 